\title{
NIELSEN FIXED POINT THEORY ON MANIFOLDS
}

\author{
ROBERT F. BROWN \\ Department of Mathematics, University of California \\ Los Angeles, CA 90095-1555, U.S.A. \\ E-mail: rfb@math.ucla.edu
}

The study of fixed points of continuous self-maps of compact manifolds involves geometric topology in a significant way in topological fixed point theory. This survey will discuss some of the questions that have arisen in this study and indicate our present state of knowledge, and ignorance, of the answers to them. We will limit ourselves to the statement of facts, without any indication of proof. Thus the reader will have to consult the references to find out how geometric topology has contributed to our knowledge in this area. But we hope this overview can supply a framework for a more detailed investigation of this important and, as we shall see, very active branch of fixed point theory.

Let $X$ be a compact connected topological manifold, possibly with boundary, and let $f: X \rightarrow X$ be a map. The fixed point set of $f$ is $\operatorname{Fix}(f)=\{x \in X: f(x)=x\}$. The principal object of study in topological fixed point theory, denoted $M F[f]$, is the minimum number of fixed points among all maps homotopic to $f$. Thus, for instance, $M F[f]=0$ means that there is a map $g$ homotopic to $f$ such that $g(x) \neq x$ for all $x \in X$.

In principle, to calculate $M F[f]$ we would have to examine the fixed point sets of every map homotopic to $f$. Fixed point theory makes use of a homotopy invariant, called the Nielsen number of $f$, the computation of which requires only a knowlege of the map $f$ itself. There are many detailed presentations of the Nielsen number; the standard texts are [3], [13] and [24]. The definition of the Nielsen number requires two concepts, the first of which is easy to describe. Define an equivalence relation on Fix $(f)$ by declaring $x, x^{\prime} \in \operatorname{Fix}(f)$ equivalent if there is a path, call it $\alpha$, in $X$ from $x$ to $x^{\prime}$ such that $\alpha$ is homotopic to $f(\alpha)$ by a homotopy keeping the endpoints fixed. The equivalence classes are called fixed point classes, often abbreviated fpc, and a fpc is represented by the symbol F. There are only a finite number of fpc for a map of a compact manifold.

The second concept required for the definition of the Nielsen number is more difficult to describe precisely, so we will limit ourselves here to an informal approach that we hope will offer some insight into how a fixed point theorist might think about this concept. A

1991 Mathematics Subject Classification: Primary 55M20.

The paper is in final form and no version of it will be published elsewhere. 
mathematically rigorous presentation can be found, for instance, in Chapter IV of [3]. Let $\mathbf{F}$ be a fpc of the map $f: X \rightarrow X$, then there is an open subset $U$ of $X$ containing $\mathbf{F}$ such that the closure of $U$ intersects $\operatorname{Fix}(f)$ only in $\mathbf{F}$. For $n$ large enough, we may embed $X$ in a euclidean space $\mathbf{R}^{n}$. For each point $x \in U-\mathbf{F}$, consider the vector in $\mathbf{R}^{n}$ from $x$ to $f(x)$. Roughly speaking, if all those vectors point in more or less the same direction, we can modify the definition of $f$ on $\mathbf{F}$ to move all those points in the direction indicated by the vectors, thus producing a map homotopic to $f$, and identical to $f$ outside of $U$, that has no fixed points on $U$. Since the fpc $\mathbf{F}$ can be eliminated in this way, $\mathbf{F}$ is said to be inessential. On the other hand, if the vectors do not all point in somewhat the same direction, the vector field on $U-\mathbf{F}$ can be thought of as "winding around" the set $\mathbf{F}$ so that it is not possible to modify $f$ by a homotopy in order to eliminate $\mathbf{F}$. Consequently, in this case, the fpc $\mathbf{F}$ is said to be essential. In a rigorous development, the amount of "winding around" of the vector field is measured by an integer, called the index of the fpc $\mathbf{F}$ and denoted by $i(\mathbf{F})$. Thus, the fpc $\mathbf{F}$ is inessential if $i(\mathbf{F})=0$ and it is essential if $i(\mathbf{F}) \neq 0$.

The Nielsen number $N(f)$ is defined to be the number of essential fpc of $f$. It can be shown that $N(f)$ is a homotopy invariant, from which it is easy to see that we must have the inequality $N(f) \leq M F[f]$.

The purpose of the Nielsen number $N(f)$, which is defined in terms of the single map $f$, is to obtain information about the minimum number $M F[f]$ that concerns the entire homotopy class of $f$. Certainly $N(f)$ is an effective tool for this purpose when $N(f)=M F[f]$. Thus, we next survey what is known about the hypotheses under which the equation $N(f)=M F[f]$ does and does not hold. We will see that it does hold, that is the Nielsen number does calculate $M F[f]$, in general, but there are important and interesting exceptions. The present survey is an updating of [4].

Since $N(f)$ usually does calculate $M F[f]$, fixed point theorists are well-motivated to discover procedures for actually calculating $N(f)$ itself. The definition sketched above does not offer much help for this task, and a rigorous presentation of the concept of essentiality does not make computation easier. However, there has been considerable progress in developing methods for computing $N(f)$ for maps of manifolds and we will survey the present state of this difficult art in the final part of the paper. For an earlier survey of the computation of the Nielsen number, see [25].

A preliminary version of this paper elicited much information and many helpful comments, and I take this opportunity to thank everyone for their contributions. I particularly thank Ross Geoghegan for his many suggestions.

\section{Wecken properties}

(a) Maps in general. Although the Nielsen number was first introduced by Nielsen in 1927 [26], its success in calculating $M F[f]$ was not demonstrated until somewhat later. In 1942, Wecken [32] proved that if $X$ is a compact, connected manifold, with or without boundary, of dimension $\operatorname{dim}(X) \geq 3$ and $f: X \rightarrow X$ is any map, then $N(f)=M F[f]$. Consequently, a manifold $X$ such that $N(f)=M F[f]$ for all maps $f: X \rightarrow X$ is said to have the Wecken property and Wecken's theorem states that a manifold $X$ has the Wecken property if $\operatorname{dim}(X) \geq 3$. 
The natural question of whether surfaces also possess the Wecken property was not settled until long after Wecken's paper was published. In 1985, Jiang [14] proved that if $X$ is a compact, connected manifold, with or without boundary, such that $\operatorname{dim}(X)=2$, then $X$ has the Wecken property if and only if the Euler characteristic $\chi(X)$ of $X$ is non-negative. Since there are only six surfaces $X$ for which $\chi(X) \geq 0$, almost all surfaces fail to have the Wecken property. Subsequent research demonstrated that, in this setting, the Wecken property fails for most surfaces in a very strong sense. A manifold $X$ is totally non-Wecken if, given $m \geq 1$, there exists a map $f_{m}: X \rightarrow X$ such that $M F\left[f_{m}\right]-N\left(f_{m}\right) \geq m$. A result of Jiang [15], extending earlier work of Kelly [18], states that if $X$ is a compact, connected manifold, with or without boundary, such that $\operatorname{dim}(X)=2$ and $\chi(X)<0$, then $\mathrm{X}$ is totally non-Wecken.

The reader will note that, unlike the previously-mentioned results, we do not mention a date for the work of Jiang and Kelly on the totally non-Wecken property. For recently published and as-yet-unpublished papers, it is difficult to assign a date in a consistent manner; moreover, in contrast to the dating of earlier publications, such a date contributes little to the reader's understanding of the development of the subject. Consequently, if, as in this case, no date is given, the reader may assume that the result is recent, specifically published since 1990 or not yet published. Of course more detailed information can be found in the list of references at the end of the paper.

The theorems of Wecken, Jiang and Kelly that we have quoted describe the Wecken property for all manifolds. (The two one-dimensional manifolds are trivially Wecken.) However, even though for $X$ a surface with $\chi(X)<0$ there are maps $f_{m}: X \rightarrow X$ such that $M F\left[f_{m}\right]-N\left(f_{m}\right) \geq m$, it could be that the maps $f_{m}$ are exceptional and, for the other maps $f: X \rightarrow X$, the Nielsen number $N(f)$ does calculate $M F[f]$, and thus the statement " $X$ is totally non-Wecken" is in this sense misleading. In any case, if there are significant classes of maps on two-dimensional manifolds for which $N(f)$ calculates $M F[f]$, it would be useful to the development of fixed point theory to know what these classes are. A map $f: X \rightarrow X$ for which $N(f)=M F[f]$ is called a Wecken map. Thus, given a Wecken map $f$, there is a map $g$ homotopic to $f$ such that $g$ has exactly $N(f)$ fixed points.

The investigation of Wecken maps is just getting under way; in fact, there is only one surface for which information is available. The surface obtained by deleting $k$ disjoint open discs from the two-dimensional sphere will be denoted by $\Delta_{k}$. The surface $\Delta_{3}$ is often called the pants surface because it is homeomorphic to a pair of pants, with one boundary circle corresponding to the waistband of the pants and the other two to the cuffs. In 1987, Kelly [17] determined $M F[f]$ for all maps of the pants surface using, for this purpose, a classification of these maps into five classes. Wagner [30] has shown that all the maps in three of the classes of the Kelly classification are Wecken whereas the other two classes contain non-Wecken maps. A natural step in the investigation of Wecken maps of surfaces would be to determine whether there are Wecken classes in some sense analogous to those three Wecken classes on the other surfaces of negative Euler characteristic.

(b) Boundary-preserving maps. For this topic, we will restrict ourselves to manifolds $X$ with nonempty boundary $\partial X$. By a boundary-preserving map of $X$ we mean a map $f: X \rightarrow X$ that takes $\partial X$ into itself. Thus, a boundary-preserving map is a map of pairs 
$f:(X, \partial X) \rightarrow(X, \partial X)$. The minimum number in the setting of boundary-preserving maps is denoted by $M F_{\partial}[f]$ and it is defined to be the minimum number of fixed points of all boundary-preserving maps of $X$ homotopic to $f$ by a boundary-preserving homotopy. That is, we consider maps $g:(X, \partial X) \rightarrow(X, \partial X)$ such that there is a map $H:(X \times I, \partial X \times I) \rightarrow(X, \partial X)$ with $H(x, 0)=f(x)$ and $H(x, 1)=g(x)$ for all $x \in X$.

Given a boundary-preserving map $f:(X, \partial X) \rightarrow(X, \partial X)$, let $\bar{f}: \partial X \rightarrow \partial X$ denote the restriction of $f$. If $X$ is a compact, connected $n$-dimensional manifold, then $\partial X$ is a compact, but not necessarily connected, $(n-1)$-dimensional manifold. If a component $\partial X_{k}$ of $\partial X$ is mapped into itself, there is a corresponding Nielsen number $N\left(\bar{f}_{k}\right)$ of the restriction $\bar{f}_{k}: \partial X_{k} \rightarrow \partial X_{k}$, that is, the number of essential fpc of $\bar{f}_{k}$. Of course if $\partial X_{k}$ is not mapped into itself by $\bar{f}$, then $\bar{f}_{k}$ has no fpc.

A boundary-preserving map $f:(X, \partial X) \rightarrow(X, \partial X)$, considered just as a map $f: X \rightarrow$ $X$, has a Nielsen number $N(f)$ defined as before: it is the number of essential fpc. Every fpc of some $\bar{f}_{k}$ is contained in some fpc of $f: X \rightarrow X$. Define the relative Nielsen number $N_{\partial}(f)$ of $f:(X, \partial X) \rightarrow(X, \partial X)$ to be the number of essential fpc of all the $\bar{f}_{k}$ plus the number of essential fpc of $f: X \rightarrow X$ that do not contain an essential fpc of any $\bar{f}_{k}$. In 1986, Schirmer [28] introduced the relative Nielsen number and proved that the inequality $N_{\partial}(f) \leq M F_{\partial}[f]$ always holds. Furthermore, she proved that if $X$ is a manifold with nonempty boundary such that $\operatorname{dim}(X) \geq 4$ and $f:(X, \partial X) \rightarrow(X, \partial X)$ is a boundary-preserving map, then $N_{\partial}(f)=M F_{\partial}[f]$.

A manifold with boundary $X$ is said to be boundary-Wecken if $N_{\partial}(f)=M F_{\partial}[f]$ for every map $f:(X, \partial X) \rightarrow(X, \partial X)$. Schirmer's theorem states that all $n$-manifolds with nonempty boundary are boundary-Wecken if $n \geq 4$. So, in this setting, the possible exceptions can occur in two dimensions: 2 and 3 .

We next discuss what is known about the boundary-Wecken property when $\operatorname{dim}(X)=$ 2 , that is, for boundary-preserving maps of surfaces with nonempty boundary. Combining results of Brown-Sanderson [5] and Kelly [19] shows that the disc $\Delta_{1}$, the annulus $\Delta_{2}$ and the Möbius band are boundary-Wecken whereas the pants surface $\Delta_{3}$ is not but it is "almost" boundary-Wecken because $M F_{\partial}[f]-N_{\partial}(f) \leq 1$ for all maps $f: \Delta_{3} \rightarrow \Delta_{3}$.

A manifold $X$ is said to be totally non-boundary-Wecken if, given $m \geq 1$, there exists a map $f_{m}:(X, \partial X) \rightarrow(X, \partial X)$ such that $M F_{\partial}[f]-N_{\partial}(f) \geq m$. Nolan [27] proved that the punctured spheres $\Delta_{k}$ are totally non-boundary-Wecken for all $k \geq 4$. Moreover, Brown-Sanderson [5] and Kelly [19] established the following result. Let $X$ be the surface with boundary obtained by deleting one or more disjoint open discs from a closed surface $K$. If $K$ is not the two-dimensional sphere or the projective plane, then $X$ is totally non-boundary-Wecken.

We have seen that all surfaces have been classified with regard to the Wecken property, but the same is not true with regard to the boundary-Wecken property for surfaces with boundary. Let $\Pi_{k}$ denote the projective plane with $k$ open discs deleted. We know that $\Pi_{1}$, the Möbius band, is boundary-Wecken, but nothing is known about $\Pi_{k}$ for $k \geq 2$. It seems reasonable to conjecture that $\Pi_{2}$ is "almost" boundary-Wecken, like the pants surface $\Delta_{3}$ (which has the same homotopy type), and that, otherwise, the $\Pi_{k}$ are totally non-boundary Wecken. 
Just as the failure of the Wecken property on surfaces leads to the study of Wecken maps, there is a corresponding notion for boundary-preserving maps. A class of maps on a surface $X$ is a boundary-Wecken class if $N_{\partial}(f)=M F_{\partial}[f]$ for every map $f:(X, \partial X) \rightarrow$ $(X, \partial X)$ in the class. The behavior of maps of the pants surface also suggests the definition of a class of maps $f:(X, \partial X) \rightarrow(X, \partial X)$ to be an almost boundary-Wecken class if there is an integer $B>0$ such that $M F_{\partial}[f]-N_{\partial}(f) \leq B$ for all maps $f$ in the class. Nolan [27] has investigated boundary-Wecken and almost boundary-Wecken classes on the $\Delta_{k}$ for $k \geq 4$ and is presently extending that investigation to other orientable surfaces with boundary.

The extent of our knowlege of the boundary-Wecken property of a manifold $X$ when $\operatorname{dim}(X)=3$ is described by two theorems. In 1986, Schirmer [28] proved that if $X$ is a 3manifold with nonempty boundary $\partial X$ such that $\chi\left(\partial X_{k}\right) \geq 0$ for every component $\partial X_{k}$ of $\partial X$, then $X$ is boundary-Wecken. Jiang, [15] proved that if $X$ is an orientable 3-manifold with nonempty boundary $\partial X$ such that $\chi\left(\partial X_{k}\right)<0$ for some component $\partial X_{k}$ of $\partial X$, then $X$ is totally non-boundary-Wecken. It is not known if nonorientable 3 -manifolds $X$ with $\chi\left(\partial X_{k}\right)<0$ for some component $\partial X_{k}$ of $\partial X$ are also totally non-boundary-Wecken, but it seems reasonable to expect that they are.

(c) Homeomorphisms. We return to the setting in which $X$ is a compact, connected manifold that may or may not have a boundary, but now we restrict ourselves to homeomorphisms $f: X \rightarrow X$. Since a homeomorphism of a manifold with nonempty boundary must map that boundary to itself, this can be viewed as a special case of the previous topic. However, for the definition of appropriate minimum number for this case, denoted $M F^{h}[f]$, we take the mimimum number of fixed points of all homeomorphisms that are isotopic, and not just homotopic, to the homeomorphism $f$. A manifold $X$ is said to have the Wecken property for homeomorphisms if $N_{\partial}(f)=M F^{h}[f]$ for all homeomorphisms $f: X \rightarrow X$, where $N_{\partial}(f)$ is defined just as before. If $X$ has empty boundary, the condition can be stated in terms of the classical Nielsen number, that is, $N(f)=M F^{h}[f]$.

A striking fact about the Wecken property for homeomorphisms is that it holds for all surfaces, in contrast to the usual Wecken property. According to Jiang-Guo [16], if $X$ is a compact, connected manifold, with or without boundary, such that $\operatorname{dim}(X)=2$, then $X$ has the Wecken property for homeomorphisms. In common with the usual Wecken property, this property also holds in sufficiently high dimensions. Kelly [20] proved that if $X$ is a compact, connected manifold without boundary such that $\operatorname{dim}(X) \geq 5$, then $X$ has the Wecken property for homeomorphisms. The methods of [20] do not apply to homeomorphisms of manifolds with boundary though it seems reasonable to expect that, in high dimensions, these would also have the Wecken property for homeomorphisms. As is often the case in geometric topology, dimensions 3 and 4 pose the greatest challenge. However, work in progress by Jiang, Wang, and Wu is establishing the Wecken property for homeomorphisms for a fairly general class of 3-manifolds.

(d) Homotopies. The two previous topics extended the fixed point theory of maps $f: X \rightarrow X$ on manifolds by restricting the type of map. Now we extend the setting from maps to homotopies. That is, consider maps $H: X \times I \rightarrow X$ and define a fixed point of a homotopy to be a solution of the equation $H(x, t)=x$ for some $t \in I$. The set of 
fixed points is denoted by $\operatorname{Fix}(H)$. A homotopy can be thought of as the one-parameter family of maps $h_{t}: X \rightarrow X$ defined by $h_{t}(x)=H(x, t)$, so this type of fixed point theory generally goes by the name one-parameter fixed point theory.

Let $P: X \times I \rightarrow X$ be the projection $P(x, t)=x$. Fixed points $(x, t)$ and $\left(x^{\prime}, t^{\prime}\right)$ are said to be equivalent if there is a path $\alpha$ in $X \times I$ such that the paths $H(\alpha)$ and $P(\alpha)$ are homotopic in $X$ by a homotopy keeping the endpoints fixed. The equivalence classes will still be called fixed point classes (fpc) and denoted F. In work of Geoghegan and Nicas [10], fixed points at the "ends" of the homotopy $H: X \times I \rightarrow X$, that is, the fixed points of $h_{0}$ and $h_{1}$, must be treated differently than the other fixed points. Let $\operatorname{Fix}_{\{0,1\}}(H)$ denote the subset of $\operatorname{Fix}(H)$ consisting of those fixed points of $H$ that are not in the same fpc as any fixed point of $h_{0}$ or $h_{1}$. Furthermore, homotopies of the given homotopy $H$ are restricted to those relative to the ends, that is, the maps $h_{0}$ and $h_{1}$ do not change during the homotopy.

Since $X \times I$ is a manifold of one dimension greater than $X$, we would expect the fixed point set of a homotopy $H: X \times I \rightarrow X$ to be infinite, so the minimum number we are concerned with is not the number of fixed points but rather the number of components of the fixed point set $\operatorname{Fix}(H)$. That is, $M F_{\{0,1\}}[H]$ is defined to be the minimum number of components of $\operatorname{Fix}_{\{0,1\}}(G)$ for all homotopies $G: X \times I \rightarrow X$ that are homotopic to $H$ relative to the ends.

To define the appropriate Nielsen number, $H$ is homotoped relative to the ends so that $\operatorname{Fix}_{\{0,1\}}(H)$ consists of isolated transverse circles. Note that if one point of a circle is in some fpc, then the entire circle is contained in that fpc. Dimovski [7] extended earlier joint work with Geoghegan [8] to define an integer homology index $\operatorname{ind}_{1}(\mathbf{F})$ and an integer mod 2 index $\operatorname{ind}_{2}(\mathbf{F})$ for any fpc $\mathbf{F}$. Denote by $N_{1}(H)$ the number of fpc of $\operatorname{Fix}_{\{0.1\}}(H)$ with nonzero ind ${ }_{1}$, by $N_{2}(H)$ the number of fpc with zero ind ${ }_{1}$ but nonzero ind $_{2}$, and define the Nielsen number to be their sum, that is, $N(H)=N_{1}(H)+N_{2}(H)$. The work of Dimovski in [7] gives an analogue of Wecken's theorem for homotopies as follows. If $X$ is a compact piecewise-linear $n$-manifold embedded in euclidean $n$-space, with $n \geq 4$, and $H: X \times I \rightarrow X$ is a homotopy, then $N(H)=M F_{\{0,1\}}[H]$.

(e) Equivariant maps. We now assume some further structure for the manifold $X$, namely, it is a smooth manifold and there is a compact Lie group $G$ acting smoothly on it. Correspondingly, we restrict attention to maps that preserve the action, that is, to $G$-maps $f: X \rightarrow X$. We also specify some subgroup $H$ of $G$ such that the Weyl group of $H$ is finite. There are two submanifolds of $X$ that we associate with the subgroup $H$. The first, denoted $X^{H}$, is the set of $x \in X$ that are fixed by all $h \in H$, that is, $h x=x$. The second, denoted $X_{H}$, consists of all $x \in X$ such that $h x=x$ if, and only if, $h \in H$. The restriction $f_{H}$ of $f$ to $X_{H}$ is a map $f_{H}: X_{H} \rightarrow X^{H}$. It is the fixed point theory of this map that concerns us in the sense that we wish to determine $M F_{G}\left[f_{H}\right]$, defined to be the minimum number of fixed points among all maps $k_{H}: X_{H} \rightarrow X^{H}$ for $k: X \rightarrow X$ a $G$-map that is $G$-homotopic to $f$.

Wong [33] defined an appropriate $G$-Nielsen number, denoted $N_{G}\left(f_{H}\right)$, and proved the following form of Wecken's theorem. If $X$ is a smooth $G$-manifold where $G$ is a compact Lie group, $H$ is a subgroup of $G$ with finite Weyl group such that $X^{H}$ is connected, $\operatorname{dim}\left(X^{H}\right) \geq 3$ and $\operatorname{dim} X^{H}-\operatorname{dim}\left(X^{H}-X_{H}\right) \geq 2$, then $N_{G}\left(f_{H}\right)=M F_{G}\left[f_{H}\right]$. 


\section{Computing $N(f)$}

(a) Convenient manifolds. In 1964, Jiang [12] demonstrated that there are important classes of manifolds with the property that, if $X$ is a manifold in one of these classes and $f: X \rightarrow X$ is any map, then the Nielsen number $N(f)$ can be calculated by means of a simple procedure. The only information about the map $f$ that is required is a description of the homomorphism $f_{\pi}$ that $f$ induces on the fundamental group of $X$ and the homomorphisms $f_{*, k}: H_{k}(X) \rightarrow H_{k}(X)$ that $f$ induces on the rational homology of $X$. The latter homomorphisms determine the Lefshchetz number $L(f)=\operatorname{Tr}\left(f_{*, 0}\right)-$ $\operatorname{Tr}\left(f_{*, 1}\right)+\ldots+(-1)^{n} \operatorname{Tr}\left(f_{*, n}\right)$ where $\operatorname{Tr}$ denotes the trace and $n=\operatorname{dim}(X)$. In all the classes covered by Jiang's result, the fundamental group $\pi_{1}(X)$ is abelian, so the image of $1-f_{\pi}: \pi_{1}(X) \rightarrow \pi_{1}(X)$ defined by $\left(1-f_{\pi}\right)[\omega]=[\omega]-f_{\pi}[\omega]$ is a normal subgroup of $\pi_{1}(X)$ and therefore the cokernel $\operatorname{Coker}\left(1-f_{\pi}\right)=\pi_{1}(X) /\left(1-f_{\pi}\right)\left(\pi_{1}(X)\right)$ is a group. Jiang proved that if $X$ is a manifold of one of the following types (1) $X=G / G_{0}$ where $G$ is a compact Lie group and $G_{0}$ is a closed, connected subgroup (2) $X$ admits an $H$-space structure (see [11] for the definition and examples) or (3) $X=S^{2 n+1} / \mathbf{Z}_{p}$ is a generalized lens space (see [29], page 88), then $L(f)=0$ implies $N(f)=0$ and $L(f) \neq 0$ implies that $N(f)$ is the order of the group Coker $\left(1-f_{\pi}\right)$.

In 1985, Anosov [1] proved that for some manifolds it was even easier to calculate $N(f)$. Suppose that $f: X \rightarrow X$ is any map where $X$ is a compact nilmanifold, that is, the quotient of a connected, simply-connected nilpotent Lie group by a cocompact discrete torsion free subgroup, then Anosov showed that $N(f)=|L(f)|$.

Keppelmann-McCord [23] extended Anosov's result to a class of solvmanifolds that includes the exponential solvmanifolds. Thus, if $f: X \rightarrow X$ is a map where $X$ is the quotient of a connected, simply-connected solvable Lie group which is exponential (the exponential map from its Lie algrebra is onto) by a cocompact discrete subgroup, then the formula $N(f)=|L(f)|$ still holds.

The Reidemeister number $R(f)$ of a map $f: X \rightarrow X$ is the number of equivalence classes of lifts of $f$ to the universal covering space of $X$ under the equivalence relation of conjugacy by covering transformations. It is generally easier to compute $R(f)$ than it is to compute the Nielsen number, so it is useful to know when the two numbers are equal. In his 1964 paper [12], Jiang considered spaces $X$ with finite fundamental groups and found conditions on them such that if $f: X \rightarrow X$ is any map, then $L(f)=0$ implies $N(f)=0$ and $L(f) \neq 0$ implies $N(f)=R(f)$. These formulas are valid, for instance, if $X$ is the quotient manifold of a finite group acting freely on an odd-dimensional sphere or if $X$ is a closed 3-manifold with finite fundamental group. Recently, Wong [34] has shown that these same formulas apply when $X$ is an orientable manifold which is the quotient space of a compact Lie group by a closed, not necessarily connected, subgroup.

There are some classes of manifolds for which there is no known formula for calculating Nielsen numbers of their selfmaps, but there are algorithms available that can, at least in prinicipal, make these calculations. There is such an algorithm for maps on any manifold with a finite fundamental group, based on techniques from [12], that is due to McCord [25] (in fact it applies to a class of spaces considerably more general than just manifolds). 
McCord also presented in [25] an algorithm for computing Nielsen numbers of maps of infrasolvmanifolds, that is, manifolds that are finitely covered by solvmanifolds.

In [21], Kelly describes two algorithms that can be used to compute the Nielsen numbers of homeomorphisms of certain 3-manifolds. One of the algorithms applies to 3-manifolds that are Seifert fiber spaces. The other can be used for homeomorphisms of certain aspherical 3-manifolds.

(b) Surfaces. We have seen in the first part of this survey that the fixed point theory of maps of compact surfaces is quite different from that of manifolds of higher dimension, so fixed point theorists are particularly concerned with finding the Nielsen numbers of such maps. McCord argues in [25] that, to a considerable extent, the level of difficulty in calculating Nielsen numbers depends on the structure of the fundamantal group of the space involved, and that the problem is particularly challenging if the group is free, or is nearly so in the sense that there is a presentation of the group with relatively few relations compared to the number of generators. Thus, from this point of view, the computation of Nielsen numbers of maps of surfaces is an especially daunting task because the fundamental group is either free or has a single relation.

On the other hand, there are tools available for studying maps of surfaces that cannot be applied to higher-dimensional manifolds. One of these tools is the Fox calculus, which Fadell-Husseini used in 1983 [9] as an aid in calculating $N(f)$ for a map $f: X \rightarrow X$ of any compact surface. Their procedure is not an algorithm, but it can be quite useful for some classes of examples. If $X$ is a closed and orientable surface, Davey-Hart-Trapp [6] have found an improvement of the Fadell-Husseini method that it is almost an algorithm; there is just one step in the process which cannot be carried out by a general rule, and even for this step there are techniques that work in many cases.

The work of Jiang-Guo [16] discussed above shows that the computation of the Nielsen numbers of homeomorphisms of surfaces tells us the minimum number of fixed points of homeomorphisms in the same isotopy class. Kelly [22] has found an algorithm for computing such Nielsen numbers. His algorithm is based on part of an algorithm of Bestvina-Handel [2] and is essentially geometric in nature.

An algebraic algorithm has been developed by Wagner [31] that applies only to surfaces with boundary, but is not restricted to homeomorphisms. However, there are other, algebraic, restrictions on the maps to which the algorithm can be applied, so the general problem of calculating the Nielsen number of any map of a surface remains open.

\section{References}

[1] D. Anosov, The Nielsen number of maps of nilmanifolds, Russian Math. Surveys 40 (1985), 149-150.

[2] M. Bestvina and M. Handel, Train tracks for surface homeomorphisms, Topology 34 (1995), 109-140

[3] R. Brown, The Lefschetz Fixed Point Theorem, Scott-Foresman, 1971.

[4] - Wecken properties for manifolds, in: Proceedings of the Conference on Nielsen Theory and Dynamical Systems, Contemp. Math. 152, 1993, 9-21. 
[5] R. Brown and B. SANDERson, Fixed points of boundary-preserving maps of surfaces, Pacific J. Math. 158 (1993), 243-264.

[6] O. Davey, E. HART and K. TrapP, Computation of Nielsen numbers for maps of closed surfaces, Trans. Amer. Math. Soc. (to appear).

[7] D. Dimovski, One-parameter fixed point indices, Pacific J. Math. 164 (1994), 263-297.

[8] D. Dimovski and R. Geoghegan, One-parameter fixed point theory, Forum Math. 2 (1990), 125-154.

[9] E. FAdell and S. Husseini, The Nielsen number on surfaces, in: Proceedings of the Special Session on Fixed Point Theory, Contemp. Math. 21, 1983, 59-98.

[10] R. Geoghegan and A. Nicas, Parametrized Lefschetz-Nielsen fixed point theory and Hochschild homology traces, Amer. J. Math. 116 (1994), 397-446.

[11] J. HARrison and J. StashefF, Families of H-spaces, Quart. J. Math. 22 (1971), 347-351.

[12] B. JIANG, Estimation of the Nielsen numbers, Chinese Math. 5 (1964), 330-339.

[13] —, Lectures on Nielsen Fixed Point Theory, Contemp. Math. 14, 1983.

[14] —, Fixed points and braids, II, Math. Ann. 272 (1985), 249-256.

[15] - Commutativity and Wecken properties for fixed points of surfaces and 3-manifolds, Topology Appl. 53 (1993), 221-228.

[16] B. JiAng and J. Guo, Fixed points of surface diffeomorphisms, Pacific J. Math. 160 (1993), 67-89.

[17] M. KELLY, Minimizing the number of fixed points for self-maps of compact surfaces, Pacific J. Math. 126 (1987), 81-123.

[18] - Minimizing the cardinality of the fixed point set for selfmaps of surfaces with boundary, Mich. Math. J. 39 (1992), 201-217.

[19] - The relative Nielsen number and boundary-preserving surface maps, Pacific J. Math. 161 (1993), 139-153.

[20] —, The Nielsen number as an isotopy invariant, Topology Appl. 62 (1995), 127-143.

[21] - Nielsen numbers and homeomorphisms of geometric 3-manifolds, Topology Proc. 19 (1994), 149-160.

[22] - Computing Nielsen numbers of surface homeomorphisms, Topology 35 (1996), 13-25.

[23] E. Keppelmann and C. MCCord, The Anosov theorem for exponential solvmanifolds, Pacific J. Math. 170 (1995), 143-159.

[24] T. Kiang, The Theory of Fixed Point Classes, Springer, 1989.

[25] C. McCord, Computing Nielsen numbers, in: Proceedings of the Conference on Nielsen Theory and Dynamical Systems, Contemp. Math. 152, 1993, 249-267.

[26] J. NiELSEN, Untersuchungen zur Topologie der geschlossenen zweiseitigen Flächen, Acta Math. 50 (1927), 189-358.

[27] J. Nolan, Fixed points of boundary-preserving maps of punctured discs, Topology Appl. (to appear).

[28] H. Schirmer, A relative Nielsen number, Pacific J. Math. 122 (1986), 459-473.

[29] E. SPAnIER, Algebraic Topology, McGraw-Hill, 1966.

[30] J. WaGner, Classes of Wecken maps of surfaces with boundary, Topology Appl. (to appear).

[31] - An algorithm for calculating the Nielsen number on surfaces with boundary, preprint.

[32] F. Wecken, Fixpunktklassen, III, Math. Ann. 118 (1942), 544-577.

[33] P. Wong, Equivariant Nielsen numbers, Pacific J. Math. 159 (1993), 153-175.

[34] —, Fixed point theory for homogeneous spaces, Amer. J. Math. 120 (1998), 23-42. 